\title{
Angiopoietin-like protein 8 (ANGPTL8)/betatrophin overexpression does not increase beta cell proliferation in mice
}

\author{
Aaron R. Cox ${ }^{1,2,3}$ - Carol J. Lam ${ }^{1,2,3}$ - Claire W. Bonnyman ${ }^{1,2,3}$ - Julia Chavez ${ }^{1,2,3}$. \\ Jacqueline S. Rios ${ }^{1,2,3}$ • Jake A. Kushner ${ }^{1,2,3}$
}

Received: 28 January 2015 / Accepted: 23 March 2015 /Published online: 28 April 2015

(C) The Author(s) 2015. This article is published with open access at Springerlink.com

\begin{abstract}
Aims/hypothesis The identification of novel targets that stimulate endogenous regeneration of beta cells would represent a significant advance in the treatment of patients with diabetes. The betatrophin hypothesis suggests that increased expression of angiopoietin-like protein 8 (ANGPTL8) induces dramatic and specific beta cell proliferation and subsequent beta cell mass expansion with improved glucose tolerance. In light of recent controversy, we further investigated the effects of ANGPTL8 overexpression on beta cell proliferation.

Methods We performed hydrodynamic tail vein injections of green fluorescent protein (GFP) or Angptl8 (also known as Gm6484) DNA in multiple cohorts of mice of different ages. We employed state-of-the-art methods to comprehensively quantify beta cell mass and proliferation, controlling for mouse age, genetic strain, source of DNA injected, Angptl8 gene expression and proliferation markers.

Results In two young and two aged cohorts of B6.129 mice, no substantial change in beta cell replication, mass or glucose homeostasis was observed following ANGPTL8 overexpression. Even in mice with extremely elevated Angptl8
\end{abstract}

Electronic supplementary material The online version of this article (doi:10.1007/s00125-015-3590-z) contains peer-reviewed but unedited supplementary material, which is available to authorised users.

Jake A. Kushner

kushner@bcm.edu

1 McNair Medical Institute, Baylor College of Medicine, Houston, TX, USA

2 Section of Pediatric Diabetes and Endocrinology, Baylor College of Medicine, Houston, TX 77030, USA

3 Pediatric Diabetes and Endocrinology, Texas Children's Hospital, Houston, TX, USA expression (26-fold increase), beta cell replication was not significantly altered. Finally, we considered mice on the ICR background exactly as studied by Melton and colleagues, and still no beta cell mitogenic effect was detected following ANGPTL8 overexpression.

Conclusion/interpretation ANGPTL8 does not stimulate beta cell replication in young or old mice.

Keywords Ageing - ANGPTL8 - Betatrophin · Lipasin · Lipid homeostasis - Pancreatic beta cell - RIFL

$\begin{array}{ll}\text { Abbreviations } \\ \text { ANGPTL8 } & \text { Angiopoietin-like protein 8 } \\ \text { EdU } & \text { 5-Ethynyl-2'-deoxyuridine } \\ \text { GFP } & \text { Green fluorescent protein } \\ \text { ITT } & \text { Insulin tolerance test } \\ \text { PPx } & \text { Partial pancreatectomy }\end{array}$

\section{Introduction}

Diabetes is associated with reduced beta cell mass leading to an absolute (type 1) or relative (type 2) insulin deficiency and hyperglycaemia $[1,2]$. Therapeutic islet transplantation suffers from loss of glucose control over time and scarcity of cadaveric islets. Stem cell generation of beta cells, while progressing, has failed to phenocopy human beta cells [3]. Consequently, the field of beta cell biology continues to focus on endogenous beta cell expansion. Normal beta cell growth occurs by self-duplication, without the contribution of specialised progenitor cells $[4,5]$. Although putative beta cell mitogens have been advanced, none has progressed as a diabetes therapy [6]. 
Multiple studies suggest circulating factor(s) stimulate beta cell proliferation in response to increased metabolic demand [7-9]. Melton and colleagues administered insulin-receptor antagonist S961 to mice in search of circulating factors [10]. Significant beta cell expansion occurred following insulin receptor antagonism, and angiopoietin-like protein 8 (ANGPTL8) was highly upregulated in liver. ANGPTL8, also known as lipasin/RIFL, was previously recognised as a potent regulator of lipid metabolism [11-13]. Melton and colleagues overexpressed ANGPTL8 in the livers of ICR mice via hydrodynamic tail vein injection, inducing a beta cell replication rate $\sim 17$-fold greater than controls, with a dramatic threefold expansion of beta cell area in 8 days and improved glucose tolerance [10]. They named the gene betatrophin, as it appeared that ANGPTL8 expanded beta cells via increased replication, furthering ANGPTL8 as a candidate therapy for diabetes. The discovery has been heralded as a major advance with the potential to improve human health [14-16]. Confusingly, ANGPTL8 levels are either increased or decreased in the serum of type 2 diabetes patients [17-19].

The actions of ANGPTL8 on beta cell generation have recently been called into question. Mice with knockout of Angptl8 (also known as Gm6484) exhibited normal glucose homeostasis [20]. Kaestner and colleagues reported that S961 administration stimulated beta cell replication in mouse but not human islets, despite a fivefold increase in Angptl8 mRNA in liver [21]. However, the failure of ANGPTL8 overexpression to induce beta cell replication in human islets in this study could have been a consequence of its use of a heterologous species model [22].

Gromada and colleagues reported that beta cell area was unaltered in Angptl8-knockout mice, even when challenged with a high-fat diet or treated with S961 [23]. They also suggest that ANGPTL8-overexpressing mice had no increase in beta cell mass, although measurements were highly variable, spanning a threefold range. Their data revealed a $\sim 30 \%$ increase in beta cell mass (the difference was not reported to be significant).

The Melton group published a reply providing data from 38 additional Angptl8-injected mice, asserting that some mice show a 'jackpot' effect on beta cell replication [24]. Although the magnitude of the response was smaller than previously observed, beta cell proliferation was still significantly above controls $(p=0.016)$. Notably, the variability of beta cell proliferation in ANGPTL8-overexpressing mice reported by the Melton group is entirely consistent with the variability of beta cell mass observed by Gromada and colleagues. Thus, Melton and colleagues propose a hypothesis in which the response to ANGPTL8 is highly variable, and some mice have a strong beta cell response. We aimed to definitively clarify the effects of ANGPTL8 on beta cell proliferation.

\section{Methods}

Mice Male B6129SF1/J mice (JAX 101043) or retired male breeders (JAX 101045) were obtained from Jackson Labs (Bar Harbor, ME, USA). Male ICR mice were obtained from Taconic (Germantown, NY, USA). Intraperitoneal GTTs were performed on day 4 after a $16 \mathrm{~h}$ fast with $2 \mathrm{~g}$ D-glucose per $\mathrm{kg}$. Insulin tolerance tests (ITTs) were performed on day 6 after a $4 \mathrm{~h}$ fast with $0.5-1.0 \mathrm{U}$ insulin (Humulin R; Eli Lilly, Indianapolis, IN, USA) per $\mathrm{kg}$. Mice were labelled continuously via the drinking water with 5-ethynyl-2'-deoxyuridine ([EdU]; 0.5 g/l; Life Technologies, Grand Island, NY, USA) from day 5 to 8 . Mice were killed 8 days after injection for beta cell morphometric analysis. Random fed serum samples collected when the mice were killed were analysed for triacylglycerol, cholesterol and VLDL. Serum insulin was measured by Mouse Ultrasensitive Insulin ELISA kit (Alpco Diagnostics, Salem, NH, USA). B6.129 mice were fed 22\% kJ from fat (catalogue number 2919; Harlan Laboratories, Houston, TX, USA). ICR mice were fed $16 \% \mathrm{~kJ}$ from fat (2920; Harlan Labs) [10].

Hydrodynamic tail vein injection B6.129 male mice of 2, 8 and 16 months of age or 7-week-old ICR male mice were used for hydrodynamic tail vein injections, as described previously [10, 25-28]. Green fluorescent protein (GFP) or Angptl8 expression plasmid DNA (generous gifts from D. Melton, Harvard University [10]), $100 \mathrm{mg}$, and sleeping beauty transposase plasmid (pCMV-SB100X; Addgene, Cambridge, MA, USA), $4 \mathrm{mg}$, were injected as $8 \%$ of body weight volume $(\mathrm{ml} / \mathrm{g})$ over $5-7 \mathrm{~s}$.

Partial pancreatectomy The splenic portion of pancreas was removed as previously $[5,29,30]$, resulting in a $\sim 50 \%$ pancreatectomy (partial pancreatectomy [PPx]).

Immunohistochemistry and morphometry Paraffin sections for pancreatic head and tail were prepared as previously described [30, 31]. The entire pancreas was sectioned every $200 \mu \mathrm{m}$, generating 8-16 sections for both head and tail pancreas. Primary antisera included guinea pig anti-insulin (catalogue number A0564, Dako, Carpinteria, CA, USA) and mouse anti-human Ki67 (catalogue number 550609; BD Biosciences, San Jose, CA, USA), followed by secondary antisera conjugated to $\mathrm{Cy} 3$ or $\mathrm{Cy} 5$ (catalogue numbers 706166-148 and 715-605-151; Jackson ImmunoResearch Laboratories, West Grove, PA, USA) and DAPI (Molecular Probes, Eugene, OR, USA). EdU was detected by Click-iT EDU Alexa Fluor 647 Imaging kit (Invitrogen, Carlsbad, CA, USA). Images were acquired using Zeiss AxioImager (Carl Zeiss MicroImaging, Thornwood, NY, USA) with Orca-ER digital camera (Hamamatsu, Middlesex, NJ, USA). Slides were imaged to quantify beta cell morphometry as previously 
described [30], using Volocity 6.1.1 software (PerkinElmer, Waltham, MA, USA).

Proliferation analysis At least 4,000 (1,700 for partial pancreatectomy) insulin ${ }^{+}$cells were counted for Ki67. At least 2,900 (987 for partial pancreatectomy) insulin ${ }^{+}$cells were counted for EdU. $\mathrm{Ki}^{+} 7^{+}$or $\mathrm{EdU}^{+}$beta cell ratios were calculated as per cent total insulin ${ }^{+}$cells.

Real-time quantitative PCR Total mRNA was extracted from liver using RNeasy Mini kit (Qiagen, Valencia, CA, USA) and cDNA prepared using High Capacity Reverse Transcription kit (Applied Biosystems, Foster City, CA, USA). Real-time quantitative (q) dual fluorescent-labelled fluorescence resonance energy transfer PCR $\left(95^{\circ} \mathrm{C} 10 \mathrm{~min}\right.$, 40 cycles $95^{\circ} \mathrm{C} 15 \mathrm{~s}$ and $60^{\circ} \mathrm{C} 1 \mathrm{~min}$ ) was performed with ABI ViiA7 real-time PCR system (Applied Biosystems) to amplify triplicate samples, comparing sample values with dilution curves. Relative gene product amounts are reported for each gene normalised to cyclophilin. Native (endogenous) Angptl8 primer/probes distinguish from total Angptl8 (exogenous plus native). Primers/probes (electronic supplementary material [ESM] Table 1) were purchased from Integrated DNA Technologies (Coralville, IA, USA). Results from a single experiment with technical replicates are averaged.
Statistics All data are mean \pm SEM, unless otherwise stated. Results were compared with Student's $t$ test (unpaired) with a Bonferroni correction when appropriate, reported as $p$ values.

Study approval The study was approved by the Baylor College of Medicine Institutional Animal Care and Use Committee.

\section{Results}

ANGPTL8 overexpression has powerful biological effects on lipids without any effect on glucose homeostasis in young B6.129 mice Melton and colleagues identified ANGPTL8 as a novel liver-derived protein that promotes beta cell growth [10]. We overexpressed ANGPTL8 via hydrodynamic tail vein injection in 2-month-old B6.129 mice (Fig. 1a). Total Angptl8 gene expression increased 4.6-fold compared with GFP controls by qPCR (Fig. 1b, ESM Table 2), equivalent to the threefold induction of Angptl8 expression induced by the insulin receptor antagonist S961 [10].

As ANGPTL8 was previously recognised as a potent regulator of lipid metabolism [11-13], we used serum lipid profiles as a biomarker of ANGPTL8 action. ANGPTL8
Fig. 1 ANGPTL8

overexpression has powerful biological effects on lipids without any effect on glucose homeostasis in young B6.129 mice. (a) Schematic indicating time course of DNA injection, GTT, ITT and killing of 2-monthold B6.129 mice. (b) Total Angptl8 gene expression analysis was performed by qPCR on liver samples, expressed as fold change from GFP-injected mice with cyclophilin used as control gene. (c-f) Measurements of random fed serum (c) triacylglycerol, (d) cholesterol, (e) VLDL and (f) blood glucose from GFP- or Angptl8-injected mice. (g) GTTs were performed on day 4 , and (h) ITTs (represented as \% of initial blood glucose) were performed on day 6, from GFP- and Angptl8injected mice. Mean \pm SEM, five animals per group. ${ }^{*} p<0.05$ and $* * * p<0.001$ vs GFP. White, GFP-injected mice; black, Angptl8-injected mice a
Hydrodynamic tail vein injection GFP or Angpt/8 DNA 2-month-old B6.129

b
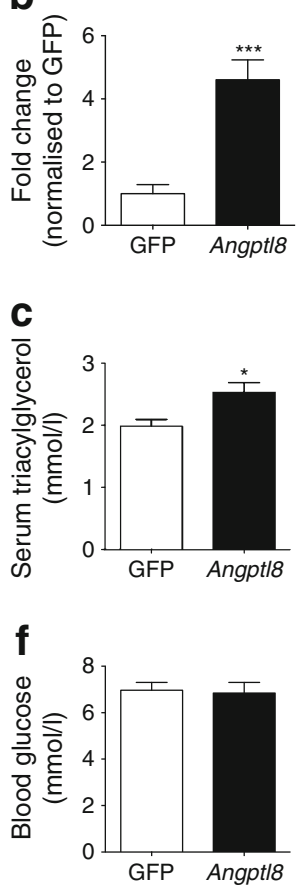

d
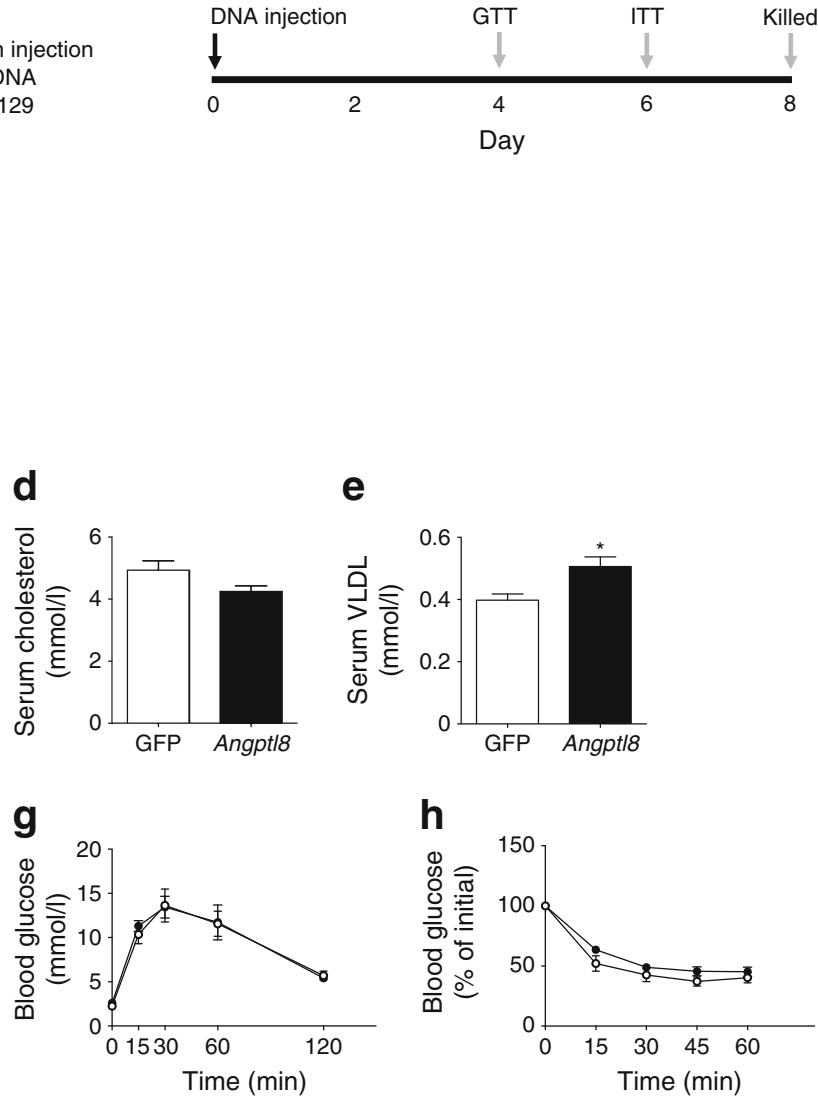
overexpression increased serum triacylglycerol and VLDL levels compared with GFP-injected controls (Fig. 1c, e), confirming biological activity of ANGPTL8. We then interrogated the impact of ANGPTL8 on glucose homeostasis. Overexpression of ANGPTL8 did not alter random fed blood glucose, glucose tolerance or insulin sensitivity (Fig. 1f-h; detailed physiological data are shown in ESM Table 3). Despite a powerful effect on lipids, ANGPTL8 had no effect on glucose homeostasis.

ANGPTL8 overexpression does not alter beta cell area in young B6.129 mice We used high-throughput quantitative imaging methods to examine beta cell content throughout the entire pancreas, sampling every $200 \mu \mathrm{m}$ to obtain total pancreas and insulin area (Fig. 2a, b). ANGPTL8 overexpression did not increase the cross-sectional insulin area or beta cell area (\% of total) compared with the GFP-injected group in young B6.129 mice (Fig. 2c, d, ESM Table 4). These results contrast with observations of a dramatic threefold increase in beta cell area over the same time period reported by Melton and colleagues [10].
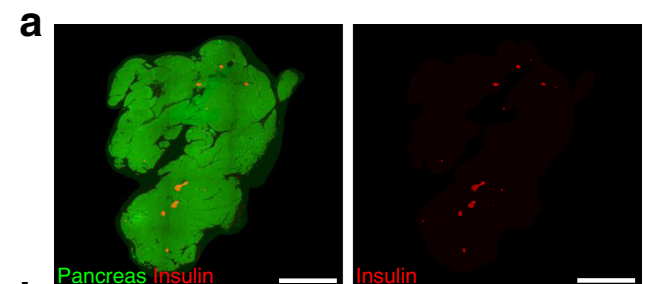

b
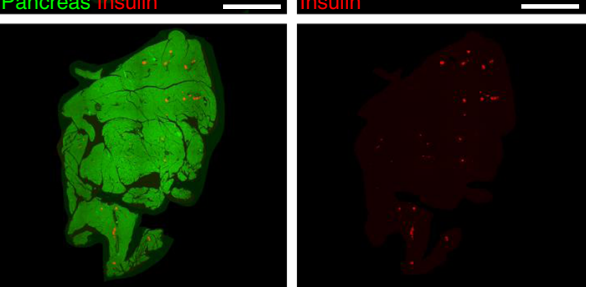

C
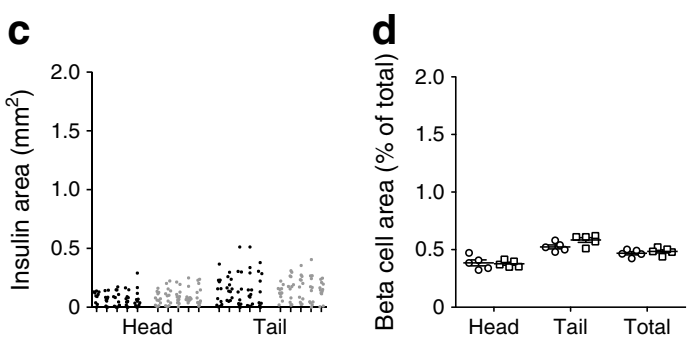

Fig. 2 ANGPTL8 overexpression does not alter beta cell area. (a, b) Representative low-power images of total pancreas (green) and total insulin area (red) for (a) GFP and (b) Angptl8 DNA-injected 2-month-old B6.129 mice. Scale bars: $2 \mathrm{~mm}$. (c) For each mouse we analysed 10-12 pancreatic sections per head or tail region, and plotted the cross-sectional insulin area for each pancreatic section as a single dot within a vertical column. Black dots, GFP; grey dots, Angptl8. (d) Beta cell area (\% of total pancreas) for the head, tail and total pancreas. Circles, GFP; squares, Angptl8. Mean \pm SEM, five animals per group
ANGPTL8 overexpression does not alter beta cell proliferation in young B6.129 mice Despite having no effect on beta cell area, ANGPTL8 may still modestly increase proliferation as recently reported [24]. Thus, we quantified beta cell proliferation via Ki67 to determine if ANGPTL8 overexpression impacts beta cell replication (Fig. 3).

Beta cells expressing Ki67 were rare in both GFP- and Angptl8-injected mice (Fig. 3b, c). In contrast, $\mathrm{Ki}^{+} 7^{+}$insulin $^{+}$ cells were dramatically increased following partial pancreatectomy ([PPx]; Fig. 3d, ESM Table 5), a well-established model of beta cell proliferation $[29,30]$. Quantification of $\mathrm{Ki} 67^{+}$beta cell proliferation showed no effect of ANGPTL8 overexpression on replication, while PPx induced a threefold increase over GFP controls (Fig. 3e).

Ki67 status gives a snapshot in time of beta cell replicative events. Thus, we also performed thymidine analogue (EdU) labelling over the final 3 days (Fig. 3a). Equally low levels of EdU incorporation were observed in beta cells of GFP and Angptl8 groups (Fig. 3f, g). ANGPTL8 overexpression had no effect on the percentage of $\mathrm{EdU}^{+}$beta cells, although proliferation was significant in the head pancreases of partially pancreatectomised mice (Fig. 3f-i, ESM Table 5). These results indicate that ANGPTL8 does not increase beta cell replication in young B6.129 mice, especially in relation to PPx, a potent inducer of beta cell proliferation.

ANGPTL8 expression in young mice We considered that our findings could be confounded by using the wrong strain/ age of mice or achieving an insufficient level of ANGPTL8 overexpression. Consequently we expanded our analysis to include a second cohort of 2-month-old B6.129 mice, two additional aged B6.129 cohorts (8- and 16-month-old), and young ICR mice (the exact strain and source of mice employed by Melton and colleagues [10]). Our initial qPCR primer/probe set measured total Angptl8 (exogenous and endogenous), thus we developed tools to quantify native (endogenous) Angptl8 gene expression.

Total Angptl8 expression was increased sixfold in the second cohort of 2-month B6.129 mice compared with GFP (Fig. 4b). This increase in expression was threefold higher than the original cohort of Angptl8-injected mice and 16-fold in absolute Angptl8 mRNA levels compared with controls for our first cohort. Native Angptl8 mRNA expression was unchanged by ANGPTL8 overexpression in both cohorts of 2-month-old B6.129 mice, indicating that the exogenous Angptl8 entirely contributed to the increase in total Angptl8 (Fig. 4b, c, ESM Table 2).

We also examined the impact of ANGPTL8 overexpression in young ICR mice. Total Angptl8 was increased by 3.2fold compared with GFP, with no change in native Angptl8 (Fig. 4b, c). Thus, we were able to overexpress Angptl8 in the exact strain of mice employed by Melton and colleagues [10]. 
Fig. 3 ANGPTL8

overexpression does not alter beta cell proliferation in young B6.129 mice. (a) Schematic indicating time course for DNA injection or PPx, with EdU labelling from day 5 to 8 . Immunostaining for (b-d) Ki67 (red), insulin (green) and DAPI (blue) for (b) GFP, (c) Angptl8 and (d) PPx groups (2-month-old B6.129 mice). (e) Quantification of beta cell proliferation measured by $\mathrm{Ki} 67^{+}$ insulin $^{+}$cells as a percentage of total insulin ${ }^{+}$cells for total pancreas. Immunostaining for (f-h) EdU (red), insulin (green) and DAPI (blue) for (f) GFP, (g) Angptl8 and (h) PPx groups (2-month-old B6.129 mice). (i) Quantification of beta cell proliferation measured by $\mathrm{EdU}^{+}$ insulin ${ }^{+}$cells as a percentage of total insulin ${ }^{+}$cells in the head, tail and total pancreas. Scale bars: $100 \mu \mathrm{m}$. Mean \pm SEM, four to five animals per group. ${ }^{* *} p<0.01$ and $* * * p<0.001$ vs GFP

\section{a}

Hydrodynamic tail vein injection or PPx GFP or Angpt/8 DNA 2-month-old B6.129

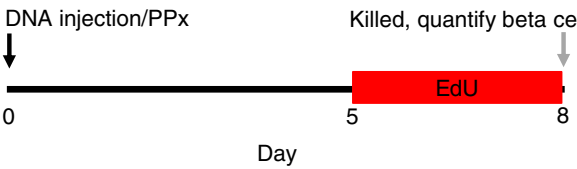

b

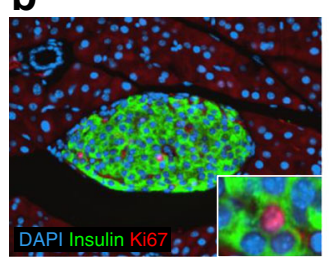

C

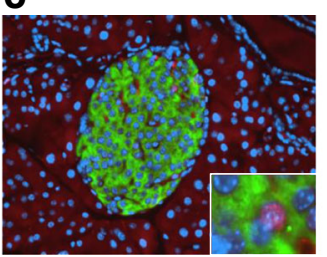

d

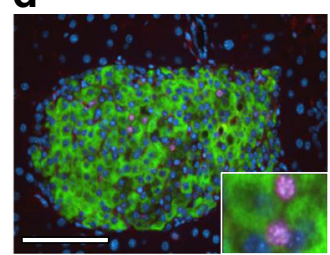

e

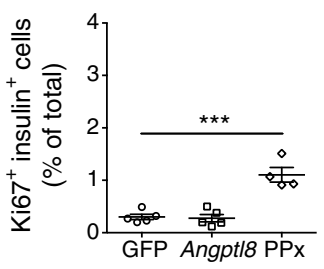

f

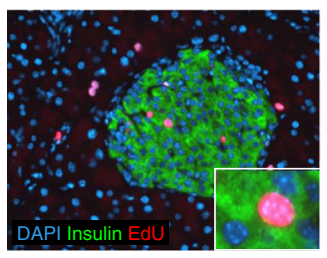

g

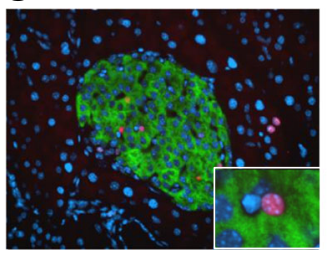

h

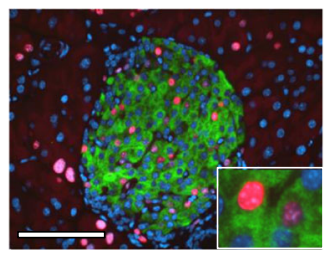

i

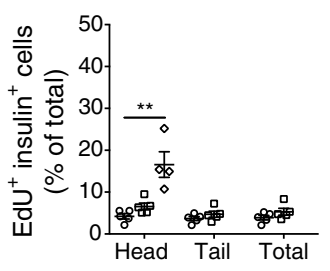

ANGPTL8 overexpression reduces native Angptl8 expression in aged mice We injected GFP or Angptl8 into 8- and 16-month-old B6.129 mice to examine the age-related impact of exogenous Angptl8 overexpression. Control aged mice exhibited tenfold increase in native Angptl8 expression compared with mice at 2 months $(p<0.05$; Fig. 4 c, ESM Table 2). Surprisingly, total Angptl8 expression was unchanged in aged mice injected with Angptl8, while native Angptl8 expression was reduced (Fig. 4b, c). This result suggests that counter-regulatory feedback loops preserve Angptl8 expression at a constant level in older mice. If such counter-regulatory loops protect Angptl8 expression levels, this could challenge the potential therapeutic utility of interventions to augment Angptl8 expression in type 2 diabetes.
ANGPTL8 overexpression does not alter beta cell area in B6.129 mice of various ages or in ICR mice We performed extensive morphometric analysis of the entire pancreas using high-throughput image processing to quantify beta cells (ESM Figs 1 and 2, ESM Table 4). Insulin area, quantified as crosssectional or cumulative total, was largely unchanged by ANGPTL8 overexpression in B6.129 mice at any age or in ICR mice (ESM Fig. 2a-h). A minimal increase in insulin area was observed in the tail of the additional 2-month-old B6.129 group (ESM Fig. 2e). Given the tiny magnitude of this difference, it likely represents the precision with which our techniques can detect small changes in biological variation as opposed to the physiological intervention of ANGPTL8 overexpression. Cumulative total pancreas area was unaltered (ESM Fig. 2i-1). 
a

Hydrodynamic tail vein injection GFP or Angptl8 DNA

2-, 8-, and 16-month-old B6.129, 2-month-old ICR

b

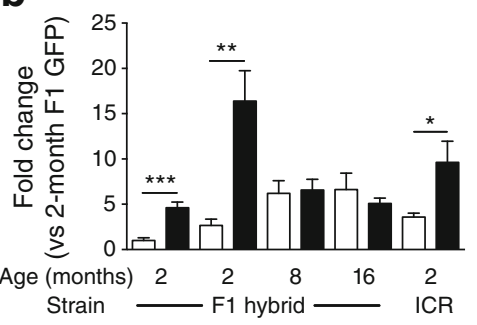

Fig. 4 Native Angptl8 increases with age and is reduced in aged mice following overexpression of exogenous Angptl8. (a) Schematic indicating time course for two cohorts of 2-, 8- and 16-month-old B6.129 mice and 2-month-old ICR mice. (b) Total and (c) native Angptl8 gene expression analysis was performed by qPCR on liver samples, expressed as fold
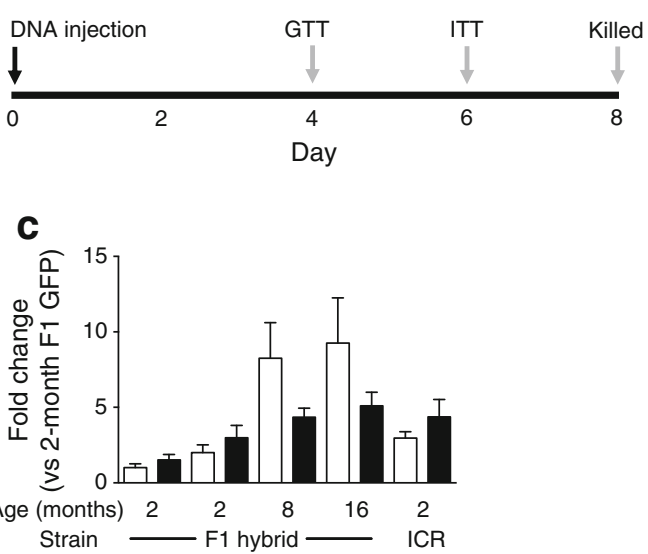

change from original 2-month-old GFP-injected B6.129 mice with cyclophilin used as control gene. The original 2-month B6.129 cohort is listed first in each graph (furthest left) followed by a second 2-month cohort. Mean $\pm \mathrm{SEM}$, five to seven animals per group. ${ }^{*} p<0.05$, $* * p<0.01$ and $* * * p<0.001$

\section{Discussion}

Although Melton and colleagues recently asserted that ANGP TL8 overexpression variably increases beta cell proliferation, we show here that ANGPTL8 overexpression in mice does not alter beta cell proliferation. We attempted to replicate the original and additional ANGPTL8 overexpression studies [10, 23, 24], including multiple cohorts of mice quantified with advanced imaging modalities. We carried out rigorous analysis of beta cell replication with both Ki67 and EdU, demonstrating that ANGPTL8 does not stimulate beta cell proliferation.

Mouse genetic strain and age could influence putative beta cell trophic actions of ANGPTL8, therefore we included an array of strains and ages. Moreover, we used the Melton Angptl8 plasmid [10]. Our initial studies in 2-month-old B6.129 mice suggested that ANGPTL8 overexpression does not expand beta cells. We then overexpressed ANGPTL8 in a second cohort of 2-month-old B6.129 mice. For reasons that are unclear, the second cohort had absolute Angptl8 levels that

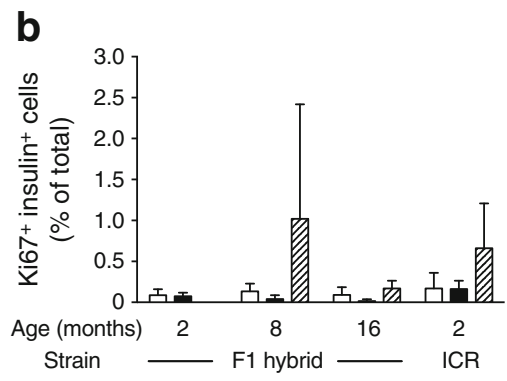

include a second group of 2-month-old B6.129 mice, 8- and 16-monthold B6.129 mice and 2-month-old ICR mice. Mean \pm SD, five to eight animals per group. White bars, GFP; black bars, Angptl8; hatched bars, PPx 
vastly exceeded other cohorts (up to 26-fold that of controls in two mice). This additional cohort therefore allowed us to rigorously test the betatrophin hypothesis, assuming higher Angptl8 expression might lead to greater stimulation of beta cell area or proliferation. However, even extreme Angptl8 expression had no effect on beta cell expansion.

Control mice at 8 and 16 months of age had higher levels of Angptl8 expression than young control mice. However, aged mice, despite their higher levels of Angptl8 expression, had far less beta cell proliferation than younger mice. Thus, even our aged controls challenge the betatrophin hypothesis.

Last, we considered the possibility that genetic strain differences could explain the lack of response in B6.129 mice. We administered Angptl8 to young ICR male mice, as performed by Melton and colleagues [10], but again found no change in beta cell proliferation. Gromada and colleagues did not match the Melton strain, age and DNA, leaving their results open to criticism that unforeseen variables could have influenced the regenerative response [23]. The reason for the discrepancy between our results and those of Melton and colleagues is not clear. However, here we have controlled for experimental conditions, matching those used by the Melton group, and conclude that ANGPTL8 overexpression has no effect on beta cell proliferation or area.

Insufficient or inappropriate sampling of beta cells could introduce unwanted variability and bias, rendering the subsequent data uninterpretable. To avoid erroneous sampling from regions of the pancreas rich in beta cells, we divided the pancreas into head and tail, sectioning every $200 \mu \mathrm{m}$ from top to bottom. This method resulted in 8-16 sections per pancreas portion, allowing for a comprehensive analysis of pancreatic beta cell area (complete raw data are in ESM Table 4).

We were concerned about the possibility of proliferating intra-islet non-beta cells contaminating our process to quantify beta cell proliferation (as previously examined [30]). As a result, we exclusively imaged islets with $\mathrm{a} \times 40$ high numerical aperture objective (0.70) to ensure beta cells (and not other cells) were correctly identified within islets. As proliferative events marked by Ki67 may be rare and highly variable, we counted at least 4, 000 beta cells per pancreas in GFP- and Angptl8-injected mice (complete raw data in ESM Table 5). Similarly, we counted over 2,900 beta cells to quantify EdU-labelled beta cells. Thus, we are confident that we have correctly assessed any potential changes in beta cell area/proliferation, and demonstrate minimal variability in beta cell area/proliferation across all cohorts, with no significant change in ANGPTL8-overexpressing mice.

Our studies included PPx as a positive control for beta cell proliferation in order to put the putative trophic effect of ANGPTL8 in the context of other known beta cell mitogenic stimuli. PPx is well documented to induce beta cell proliferation [29, 30]. As expected, PPx induced beta cell replication by three- to fivefold in young B6.129 and ICR mice. However, ANGPTL8 overexpression had no effect on beta cell replication. A regenerative response to $\mathrm{PPx}$ was maintained at 8 months of age but declined by 16 months, corroborating previous studies demonstrating an age-related decline in adaptive beta cell proliferation [29]. Even though beta cells remained responsive to regenerative stimuli at 8 months of age, no such response was observed with ANGPTL8 overexpression in mice at 8 or 16 months.

The large number of biological replicates allows us to test the 'jackpot' hypothesis recently advanced by Melton and colleagues [24], whereby some mice respond strongly to ANGPTL8 but many others do not. In this scenario, occasional mice would have extreme beta cell proliferation compared with their peers because of intrinsic differences caused by some unidentified variable. To address the possibility of a variable jackpot response, we carried out our studies in a large number of mice (28 controls and 31 experimental animals). We quantified beta cell proliferation with Ki67 (as performed by Melton and colleagues with great variability) and longterm EdU labelling to aid precise measurement of beta cell proliferation. However, no proliferative response to ANGP TL8 expression was observed, with little intrinsic variation in beta cell proliferation within mice. The largest variability occurred in the original 2-month-old B6.129 cohort; beta cell proliferation in one mouse was $0.5 \%$ with $\mathrm{Ki} 67$ while another mouse was $0.12 \%$ (ESM Table 5). Young ICR Angptl8injected mice had $\mathrm{EdU}^{+}$replication rates that peaked at $7.67 \%$ and $7.29 \%$ (identified as $325.1 \mathrm{~W}$ and $325.4 \mathrm{~W}$, respectively), which was comparable with the highest beta cell proliferation in the control GFP group $(7.17 \%$, identified as 323.3 W; ESM Table 5).

The lack of intrinsic variability in beta cell response to ANGPTL8 is in sharp contrast to the highly variable beta cell proliferation results of Melton and colleagues, and the variable beta cell mass observed by Gromada and colleagues [10, 23, 24]. The absolute values and equally small variability across all cohorts are inconsistent with the hypothesis that a subset of mice potently respond to ANGPTL8 overexpression. Instead, it seems likely that any variation previously attributed to contribute to a jackpot hypothesis might be the result of undersampling or technical differences in between cohorts (such as variable age of mice tested). Taken together, these results strongly oppose the premise that some mice respond strongly to ANGPTL8 overexpression.

A final potential confounding factor to consider is the expression level of Angptl8 necessary to induce beta cell proliferation. Previous reports did not quantify Angptl8 gene expression following hydrodynamic tail vein injection $[10,23$, 24]. Liver Angptl8 was increased by $~ 4.5$-fold in NOD severe combined immunodeficiency (SCID) mice and threefold in ICR mice following 7 days of S961 treatment and was associated with an extraordinary amount of beta cell proliferation ( $>20$ - and 12-fold) [10, 21]. These expression values are equivalent to those found in our studies, with total Angptl8 
increasing by 3.2-fold and 4.6-fold in ICR and B6.129 mice, respectively. Importantly, our second cohort of Angptl8injected mice had extremely high levels of Angptl8 expression (sixfold vs GFP and 16-fold compared with controls of our first cohort), but no increase in beta cell proliferation.

In summary, we show that ANGPTL8 overexpression does not increase beta cell proliferation. Melton and colleagues initially reported that exogenous Angptl 8 induced massive beta cell expansion by threefold within 8 days [10]. Gromada and colleagues recently reported that ANGPTL8 does not control beta cell expansion [23]. Notably, Gromada and colleagues did not quantify beta cell proliferation. Confusingly, Melton and colleagues responded that ANGPTL8 overexpression increased beta cell replication [24]. However, we provide studies in multiple cohorts of mice of various ages and genetic strains, employing the most rigorous methods in current use to quantify beta cells, finding that ANGPTL8 overexpression does not alter beta cell proliferation or expansion.

Acknowledgements The authors thank C. Blalock, L. Herrera, D. Kettlewell, B. Pekkattil, K. Rogers, K. Stephens, S. Ramirez and S. Vale of the Texas Children's Diabetes and Endocrinology Center for their administrative expertise and support. Thanks to the Baylor College of Medicine Comparative Pathology Laboratory for analysis of serum lipid profiles.

Funding This study was supported by the Robert and Janice McNair Foundation and NIH (1R01AG040110), the Pathology and Histology Core at Baylor College of Medicine with funding from the NIH (NCI P30-CA125123) and the Diabetes Research Center of the Baylor College of Medicine (DRC - P30DK079638).

Duality of interest JAK has served on the scientific advisory board of Johnson \& Johnson and currently serves on an advisory board for Lexicon. All other authors declare that there is no duality of interest associated with their contribution to this manuscript.

Contribution statement ARC conceived and designed the experiments, performed the experiments, analysed data, wrote the manuscript and approved the final version. CJL, CWB, JC and JSR performed the experiments, analysed data, wrote the manuscript and approved the final version. JAK conceived and designed the experiments, analysed data and wrote the manuscript. JAK is the guarantor of this work and has approved the final version.

Open Access This article is distributed under the terms of the Creative Commons Attribution 4.0 International License (http:// creativecommons.org/licenses/by/4.0/), which permits unrestricted use, distribution, and reproduction in any medium, provided you give appropriate credit to the original author(s) and the source, provide a link to the Creative Commons license, and indicate if changes were made.

\section{References}

1. Gepts W (1965) Pathologic anatomy of the pancreas in juvenile diabetes mellitus. Diabetes 14:619-633
2. Butler AE, Janson J, Bonner-Weir S et al (2003) Beta-cell deficit and increased beta-cell apoptosis in humans with type 2 diabetes. Diabetes 52:102-110

3. Kushner JA, MacDonald PE, Atkinson MA (2014) Stem cells to insulin secreting cells: two steps forward and now a time to pause? Cell Stem Cell 15:535-536

4. Dor Y, Brown J, Martinez OI, Melton DA (2004) Adult pancreatic beta-cells are formed by self-duplication rather than stem-cell differentiation. Nature 429:41-46

5. Teta M, Rankin MM, Long SY, Stein GM, Kushner JA (2007) Growth and regeneration of adult beta cells does not involve specialized progenitors. Dev Cell 12:817-826

6. Kulkarni RN, Mizrachi EB, Ocana AG, Stewart AF (2012) Human beta-cell proliferation and intracellular signaling: driving in the dark without a road map. Diabetes 61:2205-2213

7. Michael MD, Kulkarni RN, Postic C et al (2000) Loss of insulin signaling in hepatocytes leads to severe insulin resistance and progressive hepatic dysfunction. Mol Cell 6:87-97

8. Flier SN, Kulkarni RN, Kahn CR (2001) Evidence for a circulating islet cell growth factor in insulin-resistant states. Proc Natl Acad Sci U S A 98:7475-7480

9. El Ouaamari A, Kawamori D, Dirice E et al (2013) Liver-derived systemic factors drive beta cell hyperplasia in insulin-resistant states. Cell Rep 3:401-410

10. Yi P, Park JS, Melton DA (2013) Betatrophin: a hormone that controls pancreatic beta cell proliferation. Cell 153:747-758

11. Quagliarini F, Wang Y, Kozlitina J et al (2012) Atypical angiopoietin-like protein that regulates ANGPTL3. Proc Natl Acad Sci U S A 109:19751-19756

12. Zhang R (2012) Lipasin, a novel nutritionally-regulated liver-enriched factor that regulates serum triglyceride levels. Biochem Biophys Res Commun 424:786-792

13. Ren G, Kim JY, Smas CM (2012) Identification of RIFL, a novel adipocyte-enriched insulin target gene with a role in lipid metabolism. Am J Physiol Endocrinol Metab 303:E334-E351

14. Lickert H (2013) Betatrophin fuels beta cell proliferation: first step toward regenerative therapy? Cell Metab 18:5-6

15. Seymour PA, Serup P (2013) Bulking up on beta cells. N Engl J Med 369:777-779

16. Crunkhorn S (2013) Metabolic disorders: betatrophin boosts betacells. Nat Rev Drug Discov 12:504

17. Chen X, Lu P, He W et al (2015) Circulating betatrophin levels are increased in patients with type 2 diabetes and associated with insulin resistance. J Clin Endocrinol Metab 100:E96-E100

18. $\mathrm{Hu} \mathrm{H}$, Sun W, Yu S et al (2014) Increased circulating levels of betatrophin in newly diagnosed type 2 diabetic patients. Diabetes Care 37:2718-2722

19. Gomez-Ambrosi J, Pascual E, Catalan V et al (2014) Circulating betatrophin concentrations are decreased in human obesity and type 2 diabetes. J Clin Endocrinol Metab 99:E2004-E2009

20. Wang Y, Quagliarini F, Gusarova Vet al (2013) Mice lacking ANGP TL8 (betatrophin) manifest disrupted triglyceride metabolism without impaired glucose homeostasis. Proc Natl Acad Sci U S A 110: 16109-16114

21. Jiao Y, Le Lay J, Yu M, Naji A, Kaestner KH (2014) Elevated mouse hepatic betatrophin expression does not increase human beta-cell replication in the transplant setting. Diabetes 63:12831288

22. Stewart AF (2014) Betatrophin versus bitter-trophin and the elephant in the room: time for a new normal in beta-cell regeneration research. Diabetes 63:1198-1199

23. Gusarova V, Alexa Corey A, Na E et al (2014) ANGPTL8/ betatrophin does not control pancreatic beta cell expansion. Cell 159:691-696

24. Yi P, Park J-S, Melton DA (2014) Perspectives on the activities of ANGPTL8/betatrophin. Cell 159:468 
25. Liu F, Song Y, Liu D (1999) Hydrodynamics-based transfection in animals by systemic administration of plasmid DNA. Gene Ther 6 : $1258-1266$

26. Song YK, Liu F, Zhang G, Liu D (2002) Hydrodynamics-based transfection: simple and efficient method for introducing and expressing transgenes in animals by intravenous injection of DNA. Methods Enzymol 346:92-105

27. Yant SR, Meuse L, Chiu W et al (2000) Somatic integration and long-term transgene expression in normal and haemophilic mice using a DNA transposon system. Nat Genet 25:35-41
28. Zhang G, Budker V, Wolff JA (1999) High levels of foreign gene expression in hepatocytes after tail vein injections of naked plasmid DNA. Hum Gene Ther 10:1735-1737

29. Rankin MM, Kushner JA (2009) Adaptive beta-cell proliferation is severely restricted with advanced age. Diabetes 58:1365-1372

30. Rankin MM, Wilbur CJ, Rak K et al (2013) Beta cells are not generated in pancreatic duct ligation induced injury in adult mice. Diabetes 62:1634-1645

31. Tuttle AH, Rankin MM, Teta M, Sartori DJ, Stein GM et al (2010) Immunofluorescent detection of two thymidine analogues (CldU and IdU) in primary tissue. J Vis Exp 2166 